\author{
Takeshi Yoshida \\ John F. Boylan \\ Brian P. Kavanagh
}

\section{What do we treat when we treat ARDS?}

Received: 21 October 2015

Accepted: 11 November 2015

Published online: 14 December 2015

(C) Springer-Verlag Berlin Heidelberg and ESICM 2015

T. Yoshida · B. P. Kavanagh $(\bowtie)$

Departments of Critical Care Medicine and Anesthesia, Hospital for Sick Children, University of Toronto, Toronto, Canada

e-mail: brian.kavanagh@utoronto.ca

\section{J. F. Boylan}

Department of Anaesthesia and Intensive Care, St. Vincent's University Hospital, University College Dublin, Dublin, Ireland

Treating individual patients is a physician's primary responsibility. To do this well, the physician must identify - as far as possible — what is the patient's diagnosis, and, over time, be able to understand and apply the results of evolving research. Identifying patients with ARDS dates from Ashbaugh's report of 12 patients in 1967 (acute onset of tachypnea, hypoxemia, and loss of compliance) [1], and the initial definition of ARDS (adult respiratory distress syndrome) by Petty in 1971 [2]. The first quantitative definition, the Lung Injury Score, by Murray in 1988 assigned values to radiographic infiltrates, oxygenation, PEEP, and respiratory system compliance [3]. Twenty-five years later, the American-European Consensus Conference (AECC) re-defined ARDS, emphasizing acute onset, hypoxemia, bilateral infiltrates on chest radiograph, and the absence of heart failure [4], and this binary definition was the basis for trial entry for the next two decades. In 2012, the Berlin definition of ARDS was proposed. No definitions, however, incorporated mechanisms of injury.
Compared with the original ARDS cohort, patients with ARDS in 2015 have very different-and diversefindings at autopsy. In a recent article in Intensive Care Medicine, Lorente and colleagues question the definition of ARDS [5]. They reviewed the records of patients who died and had an autopsy, but who, while alive, fulfilled criteria for ARDS; and, they compared the clinical features of those who had diffuse alveolar damage (DAD, the histologic hallmark of ventilation-associated injury) versus those who did not. The presence of DAD on autopsy was associated with greater severity of ARDS. While this finding questions the role of a histologic 'gold standard' for diagnosis of ARDS, perhaps the first question is: 'Is there a gold standard for ARDS'?

Let us step away from ARDS and consider a diagnostic test for cancer against a histologic 'gold standard': a test with high sensitivity would miss few 'true' cancers but might include many non-cancers; in contrast, a good 'diagnostic' test would yield few false positives. To apply this reasoning in ARDS, the first step is to determine if there is a gold standard. Many believe that the gold standard for ARDS is 'DAD' $[6,7]$, and, if true, then the Berlin definition performs well as a screening test (i.e. misses few cases), but it over-diagnoses the condition (i.e. includes many who do not have positive histology).

Using limited autopsy data, Lorente et al. refer to a reference standard [5]; however, at the time of their inception, neither the AECC nor Berlin criteria for ARDS were validated against clinical data (i.e., a range of patients who might or might not have the condition, subsequently adjudicated against another method) [8]. Without a reference standard, a 'validated' diagnosis in research often consists of two clinicians' agreement on a chest X-ray (which is often poor) [9]. In clinical practice, of course, the diagnosis may be based on the judgment of a single clinician.

There are important reasons why a definition of ARDS may be useful and necessary. We believe that a definition 
of ARDS is useful for population comparisons or planning. Such information is relatively basic and concerns severity of illness and major needs for care. For example, a minimal number of variables from a large database of ICU patients could reliably identify those who developed acute respiratory failure, or hypoxemia, or who required mechanical ventilation for several days. Capturing such patients is key to planning for resources and staffing and for comparing outcomes across regions or countries. Sometimes, more specific details may not matter so much. If the purpose is to know how many patients will need an ICU bed or a mechanical ventilator, then almost any definition of ARDS would perform well.

In some settings, especially where personalized medicine is not embraced (or where expertise is limited), a definition of 'ARDS' may serve as a means of entry to a standardized treatment plan, e.g., a protocol for ARDS. In such situations, the emphasis is on the 'syndrome' as opposed to the individual patient's diagnosis, and the provision of the care will reflect a more approximate sense of the patient's condition. Nonetheless, a simplistic definition of ARDS might elevate previously low standards of care.

A major use for a definition is to determine entry into a clinical study. In some cases, an approximate definition may suffice. An extreme example might be a study testing the effectiveness of hand washing, because here the biologic characteristics of the lung disease might not matter much. In contrast, to test the effectiveness of lung recruitment one would wish to include only patients with (and, equally, exclude those without) recruitable lung. Thus, in order to be useful as a sole entry criterion, a definition of ARDS would need to incorporate the physiologic (or molecular) mechanisms under investigation.

This illustrates a major problem with clinical studies in ARDS. If histology is the gold standard for ARDS, then using the consensus definitions (AECC, Berlin) for recruiting patients into RCTs means that about $50 \%$ of those recruited probably did not have ARDS [10]. Also, even if the RCTs included only patients with ('true')
ARDS, interpretation is confounded because ARDS is a syndrome (not a disease) with multiple causes and outcomes. Thus, clinical studies contain substantial 'noise', reflecting biological complexity and reducing statistical power. Indeed, many 'negative' RCT answers may be indeterminate [11].

Syndromes are also a problem. We see many critically ill patients with 'encephalopathy'; such a label prompts a search among multiple possible explanations (e.g., thrombosis, hemorrhage, demyelination, infection, metabolic processes), each of which has a different cause, treatment and outcome. 'Encephalopathy' is never a singular diagnosis, or an indicator for a singular therapy; instead, the label signals the need for assessment and diagnosis. We cannot imagine, in the acutely injured lung, any combination of hypoxemia, PEEP and pulmonary infiltrates that would reliably predict a singular histologic appearance. However, we believe that fulfilling (any of) the consensus definitions should alert the clinician to search for a cause, and if mechanical ventilation is needed, to be aware of the dangers of ventilator-associated injury. This seems to be wise if the condition is ultimately classified as 'ARDS', or as a mimic of ARDS.

In the end, treating the patient is what matters. Any definition that directly assists patient management, or increases the yield of interpretable research, will have some use. However, we hope that future definitions of 'ARDS' will take into account the physiological and biochemical mechanisms at play; this, of course, is no simple matter.

\section{Compliance with ethical standards}

Funding Dr Kavanagh is the holder of the Dr Geoffrey Barker Chair in Critical Care Research and is funded by Operating Grants from the Canadian Institutes of Health Research. Dr Yoshida holds a RESTACOMP training award from the Research Institute, The Hospital for Sick Children, Toronto.

Conflicts of interest None of the authors have a financial conflict of interest in the subject matter of this article.

\section{References}

1. Ashbaugh DG, Bigelow DB, Petty TL, Levine BE (1967) Acute respiratory distress in adults. Lancet 2:319-323

2. Petty TL, Ashbaugh DG (1971) The adult respiratory distress syndrome. Clinical features, factors influencing prognosis and principles of management. Chest 60:233-239

3. Murray JF, Matthay MA, Luce JM, Flick MR (1988) An expanded definition of the adult respiratory distress syndrome. Am Rev Respir Dis 138:720-723
4. Bernard GR, Artigas A, Brigham KL, Carlet J, Falke K, Hudson L, Lamy M, LeGall JR, Morris A, Spragg R (1994) Report of the american-european consensus conference on ards: definitions, mechanisms, relevant outcomes and clinical trial coordination. The Consensus Committee. Intensive Care Med 20:225-232
5. Lorente JA, Cardinal-Fernández $P$, Muñoz D, Frutos-Vivar F, Thille AW, Jaramillo C, Ballén-Barragán A, Rodríguez JM, Peñuelas O, Ortiz G, Blanco J, Pinheiro BV, Nin N, Marin M, Esteban A, Thompson BT (2015) Acute respiratory distress syndrome in patients with and without diffuse alveolar damage: an autopsy study. Intensive Care Med 41:1921-1930. doi: 10.1007/s00134-015-4046-0 
6. Thille AW, Esteban A, FernandezSegoviano P, Rodriguez JM, Aramburu JA, Penuelas O, Cortes-Puch I, Cardinal-Fernandez P, Lorente JA, Frutos-Vivar F (2013) Comparison of the berlin definition for acute respiratory distress syndrome with autopsy. Am J Respir Crit Care Med 187:761-767

7. Katzenstein AL, Bloor CM, Leibow AA (1976) Diffuse alveolar damage-the role of oxygen, shock, and related factors. A review. Am J Pathol 85:209-228
8. Ransohoff DF, Feinstein AR (1978) Problems of spectrum and bias in evaluating the efficacy of diagnostic tests. N Engl J Med 299:926-930

9. Meade MO, Cook RJ, Guyatt GH, Groll R, Kachura JR, Bedard M, Cook DJ, Slutsky AS, Stewart TE (2000)

Interobserver variation in interpreting chest radiographs for the diagnosis of acute respiratory distress syndrome. Am J Respir Crit Care Med 161:85-90
10. Frohlich S, Murphy N, Boylan JF (2013) ARDS: progress unlikely with non-biological definition. Br J Anaesth 111:696-699

11. Marini JJ (2008) Lung injury—settle for a sketch or design a blueprint? Crit Care Med 36:2922-2925 